\title{
Biosorción de iones cobre con biomasa de algas y orujos deshidratados $^{(\cdot)}$
}

\author{
P. Tapia*, M. Santander**, O. Pávez**, L. Valderrama*, D. Guzmán** y L. Romero*
}

\begin{abstract}
Resumen Se realizaron experimentos de biosorción batch y en continuo para remover cobre desde soluciones acuosas usando como adsorbentes algas verdes y residuos de aceituna en condiciones vírgenes y activadas químicamente. Los resultados de la biosorción a escala batch indican que las algas presentan mayor capacidad de eliminación que los orujos, alcanzándose captaciones de cobre del orden de $96 \%$ con algas activadas con disoluciones de $\mathrm{Na}_{2} \mathrm{SO}_{4}$ bajo condiciones óptimas de las variables estudiadas. Los resultados de los ensayos en columna muestran que las algas vírgenes captan más iones cobre que las activadas con $\mathrm{Na}_{2} \mathrm{SO}_{4}$, con eficacias de eliminación del $98 \%$ durante los primeros $20 \mathrm{~min}$, con un tiempo de ruptura de $240 \mathrm{~min}$ y una saturación a los $600 \mathrm{~min}$. Al ser sometidas a un segundo ciclo de biosorción, las algas regeneradas muestran un mejor rendimiento lo que indica que pueden ser usadas en otro ciclo de eliminación.
\end{abstract}

\section{Biosorption of copper ions with biomass of algae and dehydrated waste of olives}

\begin{abstract}
They were carried out experiments of biosorción batch and in continuous to remove copper from aqueous solutions using as adsorbents green algae and olive residues under virgins conditions and chemically activated. The results of batch biosorption indicate that the algae present mayor elimination capacities than the waste of olives, with uptakes of copper of the order of $96 \%$ using activated algae with dissolution of $\mathrm{Na}_{2} \mathrm{SO}_{4}$ under the optimum conditions. The results of the columns tests show that the virgin algae permits the removal of more copper ions than the activate algae, with removal efficiency of $98 \%$ during the firth $20 \mathrm{~min}$, a breakthrough time of $240 \mathrm{~min}$ and a saturation at time of $600 \mathrm{~min}$. In the second cycle the regenerated biomass showed a best performance indicating that they can be used for another biosorption cycle.
\end{abstract}

Keywords

Biosorption; Coppers ions; Activation; Biomass.

\section{INTRODUCCIÓN}

La actividad industrial y minera arroja al ambiente metales tóxicos como plomo, mercurio, cadmio, cobre, arsénico y cromo, muy perjudiciales para la salud humana y para la mayoría de las formas de vida. Estos metales pueden detectarse en el medio ambiente en su estado elemental lo que implica que no están sujetos a biodegradación o a formación de complejos salinos por lo que en estas circunstancias no pueden ser mineralizados ${ }^{[1]}$.

Una vez emitidos, pueden permanecer en el ambiente durante cientos de años. La toxicidad de los metales pesados y su efecto en el ecosistema han generado en los últimos años un incremento considerable de estudios relacionados con la eliminación de estos elementos desde soluciones acuosas ${ }^{[2]}$.

Teniendo en cuenta los efectos que provocan los metales pesados, es evidente que se hace necesaria su eliminación de los diversos efluentes. Las industrias se ven obligadas a reducir la cantidad y la peligrosidad de sus vertidos al medio ambiente, a causa de las presiones legales, sociales y económicas $^{[2]}$.

Normalmente, son utilizados diferentes procesos para la descontaminación de efluentes que contienen

(•) Trabajo recibido el día 27 de Marzo de 2010 y aceptado en su forma final el día 2 de Julio de 2010.

* Universidad de Atacama, Departamento de Metalurgia, Copiapó-Chile, E-mail: patricia.tapia@uda.cl.

** Universidad de Atacama, Departamento de Metalurgia y CRIDESAT, Copiapó-Chile. 
metales pesados, entre ellos se pueden destacar: precipitación/ filtración, flotación, intercambio iónico, tecnología de membrana y adsorción. Sin embargo, la aplicación de estos procesos muchas veces resultan impracticables ya sea por razones económicas y/o por no ser eficientes en el tratamiento de soluciones muy diluidas ${ }^{[2]}$.

Frente a las tecnologías convencionales, el uso de sistemas biológicos para la eliminación de metales pesados en soluciones diluidas, tiene un gran potencial para conseguir mejores resultados y a un menor costo, lo cual ha levado al desarrollo de estas tecnologías en el último tiempo ${ }^{[3]}$.

Dentro de los tratamientos biológicos, la biosorción es una de las tecnologías más prometedoras para el tratamiento de aguas residuales con bajos concentraciones de metales pesados ${ }^{[45]}$. Considerando lo anterior, se torna relevante investigar y desarrollar materiales sorbentes simples y de bajo costo ${ }^{[2]}$, en este sentido, el presente trabajo tuvo como objetivo estudiar la biosorción de iones cobre mediante algas del género ulva y orujos de aceitunas, a escala batch y en columnas de relleno.

\section{DESARROLLO EXPERIMENTAL}

\subsection{Preparación y caracterización de la biomasa}

Las biomasas utilizadas en este estudio se prepararon a partir de algas verdes del género ulva y orujos de aceituna deshidratados. Las algas fueron recolectadas en el litoral de la región de Atacama (Chile) y los residuos de aceitunas (residuos agroindustriales), fueron solicitados a empresas productoras de aceite de oliva de la región de Atacama. Las biomasas fueron deshidratadas a temperatura ambiente y a $50{ }^{\circ} \mathrm{C}$ en una estufa, posteriormente se clasificaron a una granulometría $100 \%<3,3 \mathrm{~mm}$.

\subsection{Efluentes y reactivos}

Los efluentes sintéticos se prepararon disolviendo sulfato de cobre pentahidratado $\left(\mathrm{CuSO}_{4} \cdot 5 \mathrm{H}_{2} \mathrm{O}\right)$, de $99 \%$ pureza marca Fluka, en $500 \mathrm{ml}$ de agua potable (con el fin de evitar incoherencias de los resultados entre las pruebas batch y en continuo) mediante agitación a una velocidad de $500 \mathrm{rpm}$. Luego el efluente concentrado, fue disuelto en agua potable hasta alcanzar una concentración aproximada de $20 \mathrm{mg} / \mathrm{l}$. En seguida se reguló el pH del efluente $(\mathrm{pH}=5$ para evitar la precipitación de los iones de cobre) por medio de la adición de hidróxido de sodio $(\mathrm{NaOH})$ o ácido clorhídrico $(\mathrm{HCl})$ ambos de calidad analítica. El volumen final del efluente fue preparado en función de las necesidades de cada estudio, aproximadamente 151 para los ensayos batch y $100 \mathrm{l}$ para los ensayos en columnas en continuo.

Para la activación de las biomasas se usaron los reactivos sulfato de sodio $\left(\mathrm{Na}_{2} \mathrm{SO}_{4}\right)$, hidróxido de calcio $\left(\mathrm{Ca}(\mathrm{OH})_{2}\right)$, cloruro de sodio $(\mathrm{NaCl}), \mathrm{NaOH}$, agua desmineralizada y ácido sulfúrico $\left(\mathrm{H}_{2} \mathrm{SO}_{4}\right)$, además, para la desorción de los iones cobre desde la biomasa se utilizó $\mathrm{HCl}$. Todos los reactivos se prepararon con agua desmineralizada.

\subsection{Metodologías}

\subsubsection{Ensayos batch}

Los ensayos de biosorción en sistema batch se realizaron en vasos de precipitados de $1.000 \mathrm{ml}$, según condiciones preestablecidas, se mezclaron las biomasas con el efluente sintético mediante agitación mecánica y/o magnética, y empleando un agitador mecánico y/o un agitador magnético. $\mathrm{El} \mathrm{pH}$ fue ajustado con una disolución de $\mathrm{NaOH}$ o $\mathrm{HCl}$ dependiendo del $\mathrm{pH}$ requerido en cada prueba. Para la medición del $\mathrm{pH}$ se utilizó un potenciómetro marca HANNA HI 8424. Los ensayos se hicieron a temperatura ambiente $\left(21-23^{\circ} \mathrm{C}\right)$.

Una vez transcurrida la sorción de los iones cobre se procedió a la separación sólido/líquido, primero prefiltrando la solución para retener el material grueso, y luego filtrando al vacío mediante una bomba manual y papel filtro Whatman № 2, para separar el material fino de la solución. Posteriormente, se midió el pH final del líquido filtrado y se separó una alícuota de aproximadamente $100 \mathrm{ml}$ para el análisis químico de cobre. La determinación de las concentraciones iniciales y residuales de cobre se realizó mediante un espectrofotómetro de absorción atómica GBC modelo AVANTA (análisis en duplicado).

Los ensayos batch se realizaron con el fin de estudiar el efecto en la eliminación de cobre de variables tales como: $\mathrm{pH}$ inicial del efluente, tiempo de contacto y concentración del biosorbente, para posteriormente, y bajo las condiciones óptimas obtenidas en las pruebas previas (concentración de cobre $20 \mathrm{mg} / \mathrm{l}, \mathrm{pH} 5$, concentración de biosorbente $40 \mathrm{~g} / \mathrm{l}$ y tiempo de contacto $15 \mathrm{~min}$ ), examinar la influencia del tratamiento químico de los biosorbentes algas y orujos en la captación del cobre de los efluentes. 


\subsubsection{Ensayos en columnas}

En función de los resultados alcanzados en las pruebas batch se utilizaron como biosorbentes algas vírgenes y algas activadas con disoluciones de $\mathrm{Na}_{2} \mathrm{SO}_{4}$.

Los ensayos de biosorción en continuo fueron efectuados en un sistema de columnas de relleno. El equipo lo conforman tres columnas cilíndricas de material plástico de $28 \mathrm{~cm}$ de altura y $4,5 \mathrm{~cm}$ de diámetro cada una, conectadas en serie por medio de tubos de $10 \mathrm{~mm}$ de diámetro, según el esquema que se muestra en la figura 1 . Además el sistema cuenta con dos estanques adaptables a las columnas, uno de 61 para las soluciones de activación y desorción de las biomasas, y otro de 1001 para almacenar las soluciones de las pruebas de biosorción.

Para la activación se disolvió, por agitación mecánica en un depósito, una cantidad de $\mathrm{Na}_{2} \mathrm{SO}_{4}$ en 61 de agua potable hasta alcanzar una concentración de $0,1 \mathrm{~N}$. Esta disolución fue alimentada al sistema de columnas de relleno por medio de una bomba peristáltica. Antes de la activación, cada columna fue cargada previamente con $25 \mathrm{~g}$ de biomasa. Posteriormente la disolución de activación fue recirculada a través del sistema durante $24 \mathrm{~h}$. Una vez transcurrido ese tiempo, para eliminar el exceso de reactivo, las biomasas fueron sometidas a un lavado con agua potable durante $60 \mathrm{~min}$. Este procedimiento se muestra en la figura 2.

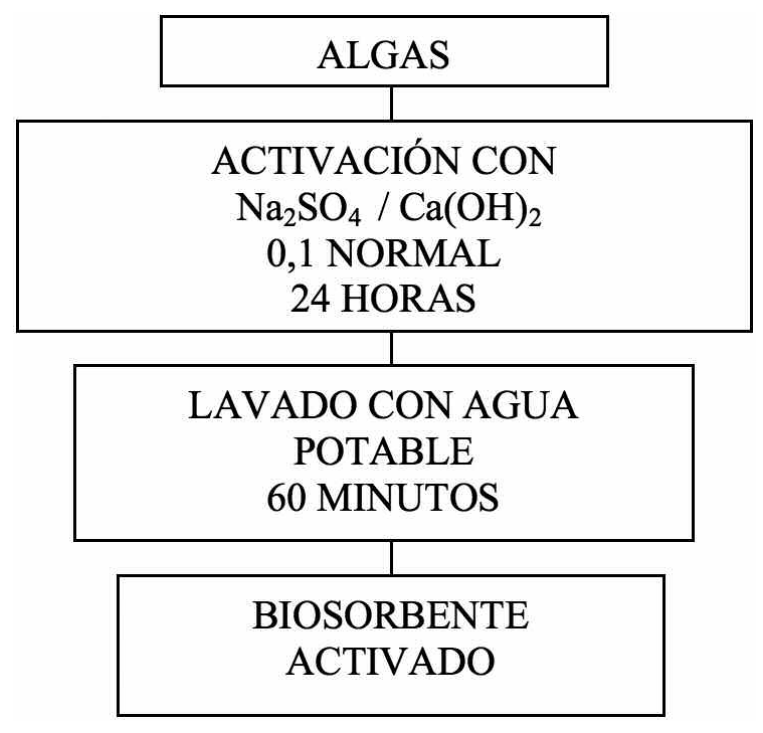

Figura 2. Esquema de tratamiento para la activación química de las biomasas usadas en los ensayos de biosorción en columnas.

Figure 2. Treatment scheme for the chemical activation of the biomasses used in the biosorption tests in columns.

El efluente fue alimentado a las columnas por medio de una bomba peristáltica marca Master Flex modelo 7518-10, ingresando en forma ascendente en la primera columna.

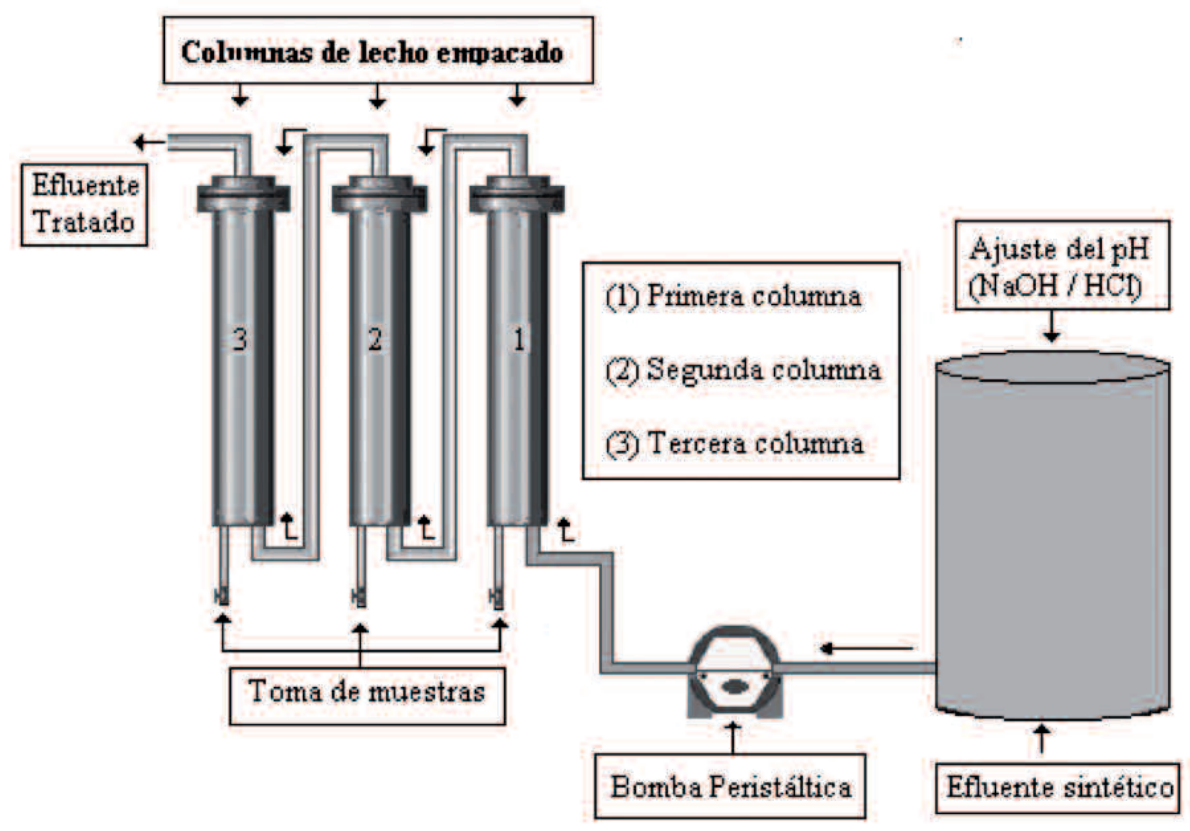

Figura 1. Sistema continúo para la biosorción de metales pesados.

Figure 1. Continuous system for the biosorption of heavy metals. 
Se realizaron los siguientes ensayos de biosorción en continuo:

- Ensayos para determinar la captación de iones cobre: Estos ensayos tuvieron como objetivo estudiar la capacidad de captación de iones cobre en el sistema de columnas, comparando la efectividad de los biosorbentes algas vírgenes y algas activadas con $\mathrm{Na}_{2} \mathrm{SO}_{4}$. Estas pruebas se desarrollaron con efluente cuya concentración inicial de cobre de $20 \mathrm{mg} / \mathrm{l}, \mathrm{pH} 5$, flujo de $50 \mathrm{ml} / \mathrm{min}$ y tiempo de biosorción de $120 \mathrm{~min}$.

- Pruebas de tiempos de ruptura y saturación: Se realizaron experiencias para obtener la curva de ruptura de los biosorbentes algas vírgenes y algas activadas con $\mathrm{Na}_{2} \mathrm{SO}_{4}$. Todos los ensayos tuvieron una duración de 22 h; además en todos ellos se mantuvo constante la concentración inicial de cobre en $20 \mathrm{mg} / \mathrm{l}$, el $\mathrm{pH} 5$, mientras que el flujo fue de $50 \mathrm{ml} / \mathrm{min}$.

- Estudios para evaluar la regeneración de las algas y su rendimiento en un segundo ciclo de biosorción: En estos ensayos las algas activadas con $\mathrm{Na}_{2} \mathrm{SO}_{4}$ usadas en las pruebas de tiempos de ruptura y saturación, fueron regeneradas para evaluar su capacidad de eliminación al ser sometidas a un segundo ciclo de biosorción. El procedimiento de regeneración consistió en recircular una disolución de $\mathrm{HCl}$ en agua potable $(1 \% \mathrm{P} / \mathrm{V})$, en el sistema de columnas de relleno por un período de $120 \mathrm{~min}$. Una vez efectuada la desorción del cobre las algas fueron lavadas con agua potable durante $60 \mathrm{~min}$. Posteriormente la biomasa fue nuevamente activada con $\mathrm{Na}_{2} \mathrm{SO}_{4}$, recirculando la disolución activadora por $12 \mathrm{~h}$, seguido de un relavado con agua potable $(60 \mathrm{~min})$. Una vez finalizado el procedimiento de regeneración del biosorbente, éste fue utilizado en un segundo ciclo de biosorción para determinar la eficacia de eliminación, el perfil de ruptura y saturación. Para estos ensayos se siguió la misma metodología y condiciones de trabajo indicadas para las pruebas de tiempos de ruptura y saturación.

- Ensayos para evaluar el efecto de la altura del lecho sobre la biosorción de iones cobre: Para ello se realizaron tres experimentos con diferentes cantidades de algas activadas con $\mathrm{Na}_{2} \mathrm{SO}_{4} 0,1 \mathrm{~N}$. Estas pruebas se efectuaron con soluciones con concentración inicial de cobre de $20 \mathrm{mg} / \mathrm{l}$, pH 5 y caudal de $50 \mathrm{ml} / \mathrm{min}$.

\section{RESULTADOS Y DISCUSIÓN}

\subsection{Ensayos batch}

La figura 3 resume los valores de captación de cobre sobre los biosorbentes algas y orujos en condiciones naturales, los resultados obtenidos indican que con ambas biomasas el incremento del $\mathrm{pH}$ provoca un aumento en la biosorción. Donde las menores eliminaciones de cobre se obtienen a $\mathrm{pH}$ altamente ácido, es bien conocido que la biosorción de metales disminuye a bajos valores de $\mathrm{pH}$ debido a las altas concentraciones de iones $\mathrm{H}^{+}$los cuales compiten con los cationes por los sitios activos disponibles en la biomasa, los resultados obtenidos sugieren que esta situación es la que ha sucedido con ambos biosorbentes dado que a partir de un $\mathrm{pH}$ inicial $2 \mathrm{el} \mathrm{pH}$ final se eleva a 3,6 y 3,1 para algas y orujos respectivamente dejando de manifiesto la disminución de la concentración de protones.

La dependencia de la remoción de metales por el $\mathrm{pH}$ esta relacionada con los grupos funcionales presentes en las paredes celulares de la biomasa y con la química de metales en solución, es por esto que es importante determinar las especies predominantes en función del $\mathrm{pH}$, en la figura $4^{[6]}$ se observa el diagrama de predominancia de especies cobre para una concentración de $20 \mathrm{mg} / \mathrm{l}$ en medio acuoso. El diagrama muestra que a $\mathrm{pH}$ inferiores a 5,5 el metal se encuentra en su estado iónico $\left(\mathrm{Cu}^{+2}\right)$, mientras que en el rango de 5,5 a 10 coexisten las especies iónicas $\mathrm{Cu}^{+2}, \mathrm{Cu}(\mathrm{OH})^{+}$y $\mathrm{Cu}(\mathrm{OH})_{2}$, a $\mathrm{pH} 6$ la solubilidad del cobre es de $20 \mathrm{mg} / \mathrm{l}$ por lo que a este $\mathrm{pH}$ todo el cobre se encuentra en solución, mientras que a $\mathrm{pH}$ 8 existe presencia de hidróxido cobre precipitado

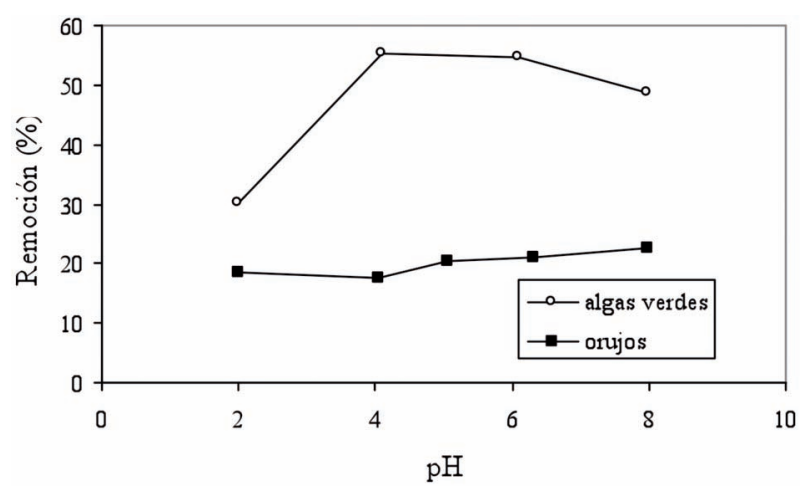

Figura 3. Efecto del pH sobre la eliminación de iones cobre en algas verdes y orujos (desechos de aceitunas).

Figure 3. $\mathrm{pH}$ effect on the removal of copper ions in biomass of algae and orujos (waste of olives). 


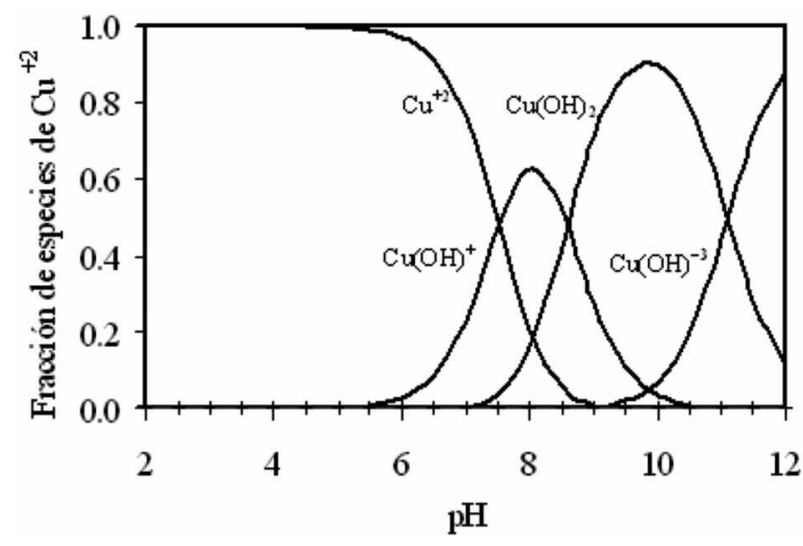

Figura 4. Distribución de especies de $\mathrm{Cu}^{+2}$ en función del $\mathrm{pH}$ para una concentración inicial de $20 \mathrm{mg} / \mathrm{l}$.

\section{Figure 4. Distribution of $\mathrm{Cu}^{+2}$ species as function of $\mathrm{pH}$ for an initial concentration $20 \mathrm{mg} / \mathrm{l}$.}

debido a que la solubilidad es de $0,05 \mathrm{mg} / \mathrm{l}$ la cual es inferior a la concentración según el diagrama de especiación de la figura 4, la aparición de este precipitado se inicia a pH 7. El cambio de especiación del cobre no es el responsable del aumento que presenta la biosorción a partir del $\mathrm{pH} 2$, esto implica que los grupos funcionales y el estado iónico en el rango de $\mathrm{pH}$ considerado determinan el grado de biosorción.

Las máxima eliminación de cobre usando orujos se consigue a $\mathrm{pH} 8$, mientras que con las algas se alcanza en el rango de $\mathrm{pH} 4$ a 6 , presentando éstas un mejor rendimiento, por lo tanto para examinar el potencial de biosorción de las biomasas y para asegurar que el cobre se encuentre en su estado iónico durante la biosorción fue seleccionado el $\mathrm{pH} 5$ para las restantes pruebas contempladas en este estudio.

Las algas contienen grupos funcionales tales como grupos carboxílico, sulfonato, hidroxilo, fosfonato, amino, pero según la literatura el grupo más importante en la adsorción de iones metálicos es el grupo carboxílico ${ }^{[7-13]}$, grupo que igualmente se encuentra presente en los orujos, este grupo funcional actúa como intercambiador iónico en la eliminación de cobre según la siguiente reacción:

$$
2(-\mathrm{COOH})+\mathrm{Cu}^{+2}=\left(-\mathrm{COO}_{2} \mathrm{Cu}\right)+2 \mathrm{H}^{+}
$$

De acuerdo a los resultados logrados en este estudio se atribuye al grupo carboxílico presente en las algas así como en los orujos la máxima recuperación de cobre alcanzada en el rango de $\mathrm{pH}$ entre 4 y 5 , y debido a los niveles de eliminación de cobre alcanzados se puede indicar que el biosorbente presenta una baja concentración de este grupo funcional.
El descenso que presentó la eliminación de cobre con las algas a $\mathrm{pH}$ superiores a 6, puede estar vinculado a que sobre este $\mathrm{pH}$ comienzan a desaparecer los iones $\mathrm{Cu}^{+2}$, apareciendo iones $\mathrm{Cu}(\mathrm{OH})^{+}$, los cuales reaccionan con los grupos aminos generando precipitados coloidales que se mantienen en solución. No fueron considerados $\mathrm{pH}$ superiores a 8 debido a la precipitación del cobre como $\mathrm{Cu}(\mathrm{OH})_{2}$.

En la literatura se han publicado una gran cantidad de artículos que muestran el efecto del $\mathrm{pH}$ en la adsorción de iones cobre en diferentes materiales adsorbentes orgánicos (cáscaras de avellanas ${ }^{[14]}$, corteza de álamos de bosque ${ }^{[15]}$, desechos de areca ${ }^{[16]}$ cáscaras de nueces, avellanas y almendras ${ }^{[17]}$, residuos de manzanas $\left.{ }^{[18]}\right)$, los rangos de $\mathrm{pH}$ informados donde ocurren los valores máximos de adsorción de cobre varían entre 4,5 a 7,5 los cuales son concordantes con los resultados obtenidos en el presente trabajo.

En la eliminación de iones cobre con algas se han publicado diferentes artículos. Yu and Kaewsarn ${ }^{[19]}$ trabajando con biomasa de algas marinas Durvillaea potatorum, encontraron que el rango de $\mathrm{pH}$ óptimo para la máxima adsorción de iones cobre es 4,1 a 5,4. Un decrecimiento en el $\mathrm{pH}$ de la solución resultó en una disminución de la capacidad de adsorción de la biomasa. Kratochvil and Volesky ${ }^{[20]}$ trabajando con aguas ferruginosas que contenían cobre señalan que la biosorción de cobre mediante biomasa de alga Sargassum aumenta con el incremento del $\mathrm{pH}$ del agua residual, encontrándose que el rango de $\mathrm{pH}$ óptimo para la biosorción de iones cobre se encuentra entre 4 y 5 . Davis et al. ${ }^{[7]}$ en una revisión realizada sobre la biosorción de metales pesados en algas marrón oscuro muestran que el pH óptimo para la adsorción de iones cobre en diferentes algas de ese tipo se encuentra en $\mathrm{pH} 4,5$. Basha et al..$^{[8]}$ indican que la máxima captación de cobre (alrededor de $61 \%$ ) se produce en $\mathrm{pH}$ igual a 6 al utilizar la especie Cystoseira como biosorbente de iones de cobre y níquel. Por otro lado, los resultados alcanzados por Deng et al..$^{[21]}$ al remover cobre utilizando Cladophora fascicularis, reportaron que en el rango de $\mathrm{pH}$ entre 4 y 5 se logran las mayores eliminaciones. Ellos indican que los grupos carboxílicos presentes en estas algas juegan un rol principal en la capacidad de adsorción de las algas. En este mismo contexto, Murphy et al. ${ }^{[9]}$ quienes estudian la adsorción de iones cobre utilizando algas marrón oscuro, verde y roja, señalan que en la determinación de la adsorción máxima de iones cobre sólo se puede considerar hasta $\mathrm{pH}$ 6, ya que a $\mathrm{pH}$ superiores a éste se produce la precipitación del cobre formándose hidróxido de cobre insoluble. Estos investigadores indican que en $\mathrm{pH}$ 2, la adsorción observada puede deberse a la presencia de 
grupos sulfonatos que están disociados a este $\mathrm{pH}$, mientras que en $\mathrm{pH}$ 3,5 - 5 los grupos carboxílicos generan carga negativa en la superficie de las algas lo que produciría una atracción electrostática entre los cationes de cobre y la superficie de las algas, pudiéndose atribuir a este fenómeno la responsabilidad de la biosorción del metal.

La máxima captación de cobre alcanzada por

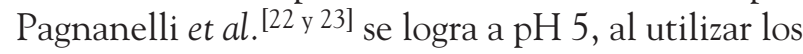
residuos de oliva (orujos).

Con el fin de decidir si un determinado biosorbente puede ser empleado en la extracción de metales, es importante conocer las condiciones en donde el fenómeno de biosorción presenta su mejor rendimiento, en cada sistema metal-biosorbente se establecen equilibrios específicos y el tiempo en el que logra alcanzar el equilibrio dependerá fundamentalmente de la naturaleza de esta relación. El tiempo en donde se logra la captación máxima permitirá además identificar el o los posibles mecanismos involucrados en la eliminación de metales.

La etapa controlante de una reacción de biosorción esta relacionada con la cinética del proceso, la cual puede ser analizada a través de modelos de reacciones de primer orden, segundo orden, pseudos-segundo orden, etc.

El efecto del tiempo de contacto en el proceso de biosorción de cobre se muestra en la figura 5 . Los resultados obtenidos muestran que la retención de cobre por ambas biomasas se produce en dos fases: la primera de forma muy rápida durante los primeros $5 \mathrm{~min}$, y la segunda en la cual se aprecia una cinética lenta, la fase rápida se debe probablemente a la disponibilidad de sitios activos en la biomasa, y con la ocupación gra-

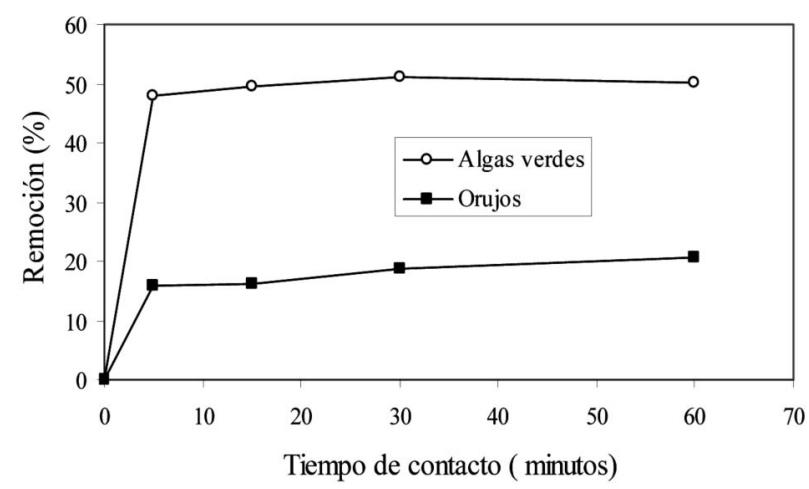

Figura 5. Efecto del tiempo de contacto en la eliminación de iones cobre sobre algas verdes y orujos (desechos de aceitunas).

Figure 5. Time of contact effect on the removal of copper ions in biomass of algae and orujos (waste of olives). dual de estos sitios la velocidad disminuye, y de esto se puede presumir que existe más de un mecanismo presente en la extracción de iones cobre. Al respecto no se puede indicar el orden de la reacción que ocurre antes de los $5 \mathrm{~min}$ posterior a este tiempo, y por la lenta cinética que se observa, se puede suponer la ocurrencia de una reacción de orden cero. Las algas presentan claramente el mejor rendimiento para el rango de tiempo comprendido entre los 5 y $60 \mathrm{~min}$. Se observa que $15 \mathrm{~min}$ es un tiempo suficiente para alcanzar el equilibrio bajo las condiciones experimentales aplicadas, con eliminaciones de cobre de $50 \%$ y $16,2 \%$, para las algas y orujos respectivamente, ambos en condiciones vírgenes.

La literatura muestra en general que las cinéticas de adsorción de iones cobre en diferentes materiales adsorbentes es rápida, alcanzándose posteriormente una adsorción estacionaria o de equilibrio debido posiblemente a la saturación ${ }^{[14-18]}$. El tiempo en que se alcanza una adsorción estacionaria es variable y según datos publicados para iones cobre utilizando diferentes biosorbentes, puede alcanzarse entre los 15 a 120 min [15-19, 8, 21, 24 y 25], e incluso después de

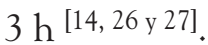

Con el objeto de determinar la mínima cantidad de biosorbente necesaria para alcanzar la máxima eliminación de cobre se realizaron experimentos con concentraciones de biosorbente entre 10 a $80 \mathrm{~g} / \mathrm{l}$, de acuerdo a los resultados que se muestran en la figura 6 , se aprecia que el porcentaje de eliminación del metal aumenta con el incremento de la concentración de ambos biosorbentes. Sin embargo, a medida que se incrementa la concentración de biomasa disminuye la capacidad de biosorción, desde 0,375 a 0,084 mg/g,

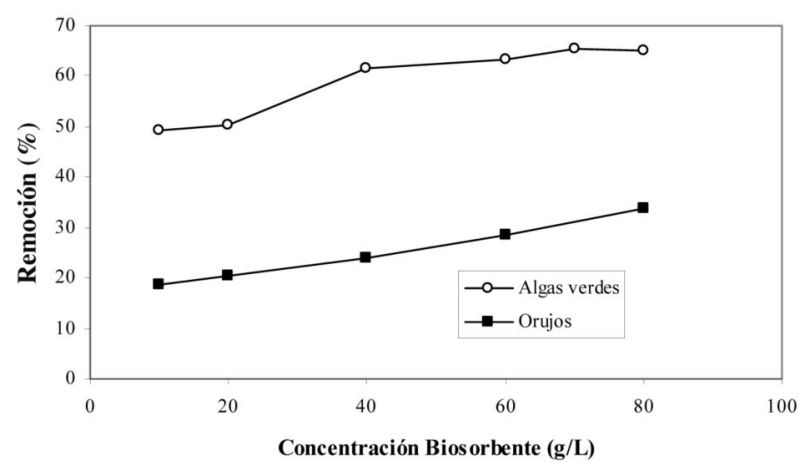

Figura 6. Efecto de la concentración del biosorbente en la eliminación de iones cobre sobre algas verdes y orujos (desechos de aceitunas).

Figure 6. Concentration biosorbente effect on the removal of copper ions in biomass of algae and orujos (waste of olives). 
debido a que aumenta la cantidad de sorbente empleado para tratar un mismo volumen de efluente. Los mecanismos que tienen lugar en un proceso de biosorción determinado son la acumulación extracelular (microprecipitación) ó la acumulación en superficie celular (complejación, adsorción física e intercambio iónico ${ }^{[28]}$. En muchas ocasiones es difícil explicar el/los mecanismos que tienen lugar en un proceso de biosorción determinado. Generalmente se considera que en la biosorción de metales pesados pueden aparecer simultáneamente más de uno de los mecanismos. Las algas y los orujos presentan en la biosorción el comportamiento de un intercambiador iónico ${ }^{[29 \text { y } 30]}$.

Los bajos incrementos en la eliminación de cobre, a medida que se aumenta la cantidad de biomasa se puede atribuir a los efectos que provocan en el proceso de intercambio iónico los iones $\mathrm{Ca}^{+2} \mathrm{y} \mathrm{Mg}^{+2}$, presentes en el agua potable, utilizada en la preparación de las soluciones de cobre, ocupando estos iones los sitios activos disponibles una vez que la concentración de cobre en la solución disminuye. En el presente estudio se seleccionó una concentración de biosorbente igual a $40 \mathrm{~g} / \mathrm{l}$ como óptima, alcanzándose eliminaciones, a partir de soluciones con $20 \mathrm{mg} / \mathrm{l}$ de cobre y pH 5, de $62 \%$ y $24 \%$ utilizando algas y orujos vírgenes, respectivamente.

En la literatura se señala que un aumento en la concentración del biosorbente incrementa la eliminación de los iones cobre, lo cual se atribuye a que habría una mayor área superficial y una mayor cantidad de sitios activos $^{[15-17]}$. Estudios realizados utilizando algas Lobophora variegata logran captaciones de $\mathrm{Cd}^{+2}$ y $\mathrm{Pb}^{+2}$ superiores a 90 \% cuando la razón sólido/líquido fue de $2 \mathrm{~g} / \mathrm{l}^{[11]}$, y en los estudios efectuados por Luo et al. las captaciones para el plomo fluctuaron entre 63 a $95 \%$ para concentraciones del biosorbente entre 0,5 a $5 \mathrm{~g} / \mathrm{l}$ ${ }^{[13]}$. Grimm et al. ${ }^{[25]}$ trabajando con diferentes concentraciones iniciales de iones cobre $(5,10$ y $20 \mathrm{mg} / \mathrm{l})$ encontraron que con una dosis de $0,2 \mathrm{~g} / 100 \mathrm{ml}$ de alga se remueve más del $90 \%$ de los iones cobre desde la solución, mientras que Basha et al. ${ }^{[8]}$ obtuvieron la máxima de sorción de cobre cercana a 57 \% a la más baja concentración de biomasa estudiada $(1,0 \mathrm{~g} / \mathrm{l})$, mostrando que existe pérdida de efectividad de la captación a altas concentraciones del biosorbente. Al usar biosólido para eliminar cobre desde soluciones acuosas Sarioglu et al..$^{[24]}$ muestran que las captaciones no son alteradas significativamente para rangos de concentraciones del biosorbente entre 3 a $9 \mathrm{~g} / \mathrm{l}$. Por otra parte, Vegliò et al. ${ }^{[31]}$ estudian a diferentes $\mathrm{pH}$ el efecto de la concentración de residuos de aceitunas molidos en dosis de 1 a $100 \mathrm{~g} / \mathrm{l}$ sobre la concentración final de cobre en la solución, obteniéndose una disminución desde 30 a $8 \mathrm{mg} / \mathrm{l}$ al incrementarse la dosis del biosorbente. Ellos señalan que en $\mathrm{pH}$ 5,5 se obtienen elimi- naciones de cobre de $20 \%, 52 \%$ y $85 \%$ al utilizar 1 , 10 y $100 \mathrm{~g} / \mathrm{l}$ de estos residuos respectivamente.

Con el fin de mejorar el desempeño de ambos biosorbentes estos fueron sometidos a diferentes tratamientos químicos, las figuras 7 y 8 muestran la capacidad de sorción de cobre y el $\mathrm{pH}$ final del efluente logrados con la algas y los orujos respectivamente, en ambos casos los niveles de eliminación de cobre fueron superiores a los alcanzados en los ensayos previos, donde los mejores resultados se logran activando las biomasas con $\mathrm{Na}_{2} \mathrm{SO}_{4}$, llegando a duplicar la eliminación de cobre con los orujos, mientras que para las algas las remociones de cobre superan el 96 \%. Este comportamiento puede deberse al hecho que el pretratamiento con este reactivo las algas y los orujos presentaron una mayor captación de iones $\mathrm{Na}^{+}$, que posteriormente

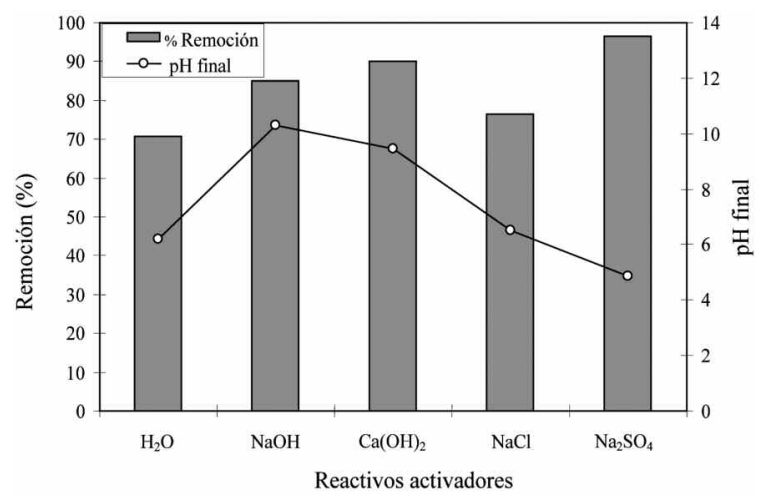

Figura 7. Efecto de la activación química de las algas sobre la eliminación de iones cobre. Figure 7. Chemical activation effect algae on the
removal of copper ions.

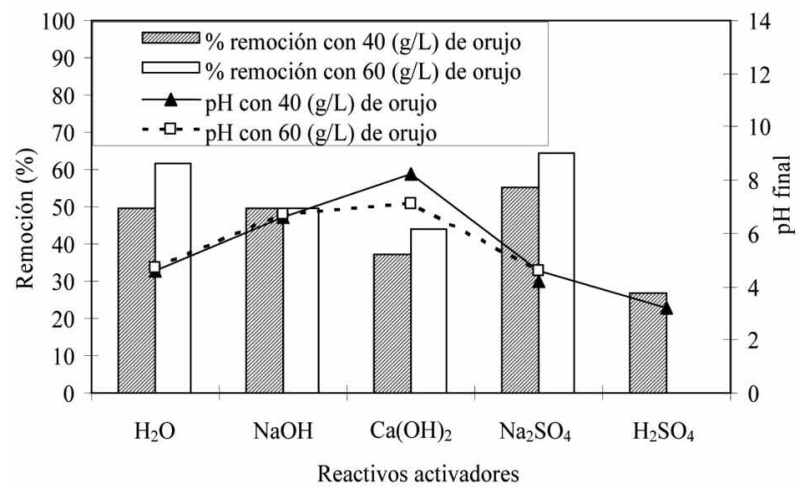

Figura 8. Efecto de la activación química de los orujos (desechos de aceitunas) sobre la adsorción de iones cobre.

Figure 8. Chemical activation effect orujos (waste of olives) on adsorption of copper ions. 
favorecieron el intercambio de iones sodio por iones cobre, cabe destacar además que al activar los biosorbentes con este reactivo el $\mathrm{pH}$ residual no presenta cambios significativos, mientras que en todos los otros casos el $\mathrm{pH}$ final alcanza valores superiores a 6 lo que conlleva a suponer que las remociones obtenidas tienen relación con la precipitación del cobre como $\mathrm{Cu}(\mathrm{OH})_{2}$.

Debido a los rendimientos inferiores en la eliminación de cobre con el orujo, se realizaron pruebas adicionales aumentándose la concentración de éste a $60 \mathrm{~g} / \mathrm{l}$, donde por el nulo efecto en el proceso, la activación con $\mathrm{H}_{2} \mathrm{SO}_{4}$ no fue considerado en el nuevo set de pruebas, lográndose aumentar la eliminación de cobre a valores cercanos a $62 \%$ cuando este biosorbente fue activado con $\mathrm{Na}_{2} \mathrm{SO}_{4}$, Díaz et al. ${ }^{[32]}$ al pretratar algas con los mismos reactivos activadores reportan eliminaciones de fierro, aluminio y manganeso superiores a $80 \%$, mientras que en la biosorción de iones $\mathrm{Cu}^{+2}, \mathrm{Zn}^{+2}$ y Cd $\mathrm{Cd}^{+2}$ efectuada con lodo activado, por Sheng-lian et al. ${ }^{[33]}$ consiguen eliminaciones para el cobre similares a las alcanzadas en este estudio. Basha et al.$^{[8]}$ también reporta resultados positivos del efecto en la eliminación de iones cobre y níquel al efectuar la modificación química del biosorbente. Ensayos orientados a evaluar la capacidad de captación de plomo con algas marrón oscuro pretratadas químicamente muestran que sólo una de las tres modificaciones químicas realizadas presenta un aumento con respecto al alga cruda ${ }^{[13]}$. Lodeiro et al. ${ }^{[34]}$ lograron mejorar las captaciones de cadmio (II) con cinco modificaciones químicas del biosorbente, mientras que otros dos tratamientos efectuados presentan resultados inferiores a los alcanzados con la especie sin tratamiento. Otros investigadores ${ }^{[35]}$ al realizar pruebas utilizando como biosorbente orujos de aceituna sin tratamiento reportan captaciones de cobre igual a $0,043 \mathrm{mmol} / \mathrm{g}$, sin embargo cuando el biosorbente fue tratado con peróxido de hidrógeno $\left(\mathrm{H}_{2} \mathrm{O}_{2}\right)$ la captación es igual a $0,056 \mathrm{mmol} / \mathrm{g}$, obteniendo una captación igual a $0,09 \mathrm{mmol} / \mathrm{g}$ cuando este mismo biosorbente fue tratado con ácido fosfórico $\left(\mathrm{H}_{3} \mathrm{PO}_{4}\right)$, valores que resultan ser similares a los alcanzados con los orujos tratados con diferentes agentes químicos en este estudio $(0,0043 \mathrm{mmol} / \mathrm{g})$. La aplicación bajo diferentes condiciones del ácido nítrico como agente activador de orujos de aceituna fue investigada por Pagnanelli et al. [36] quienes reportan aumentos significativos en la biosorción de cobre.

En función de los rendimientos alcanzados en las pruebas de activación química se seleccionaron las algas verdes como biosorbente para realizar los ensayos de biosorción en columnas, donde se evaluó su comportamiento en condiciones virgen y activada con $\mathrm{Na}_{2} \mathrm{SO}_{4}$.

\subsection{Ensayos en columnas}

\subsubsection{Captación de cobre y curva de ruptura}

La figura 9 muestra la concentración residual de cobre en el efluente en función del tiempo de biosorción,

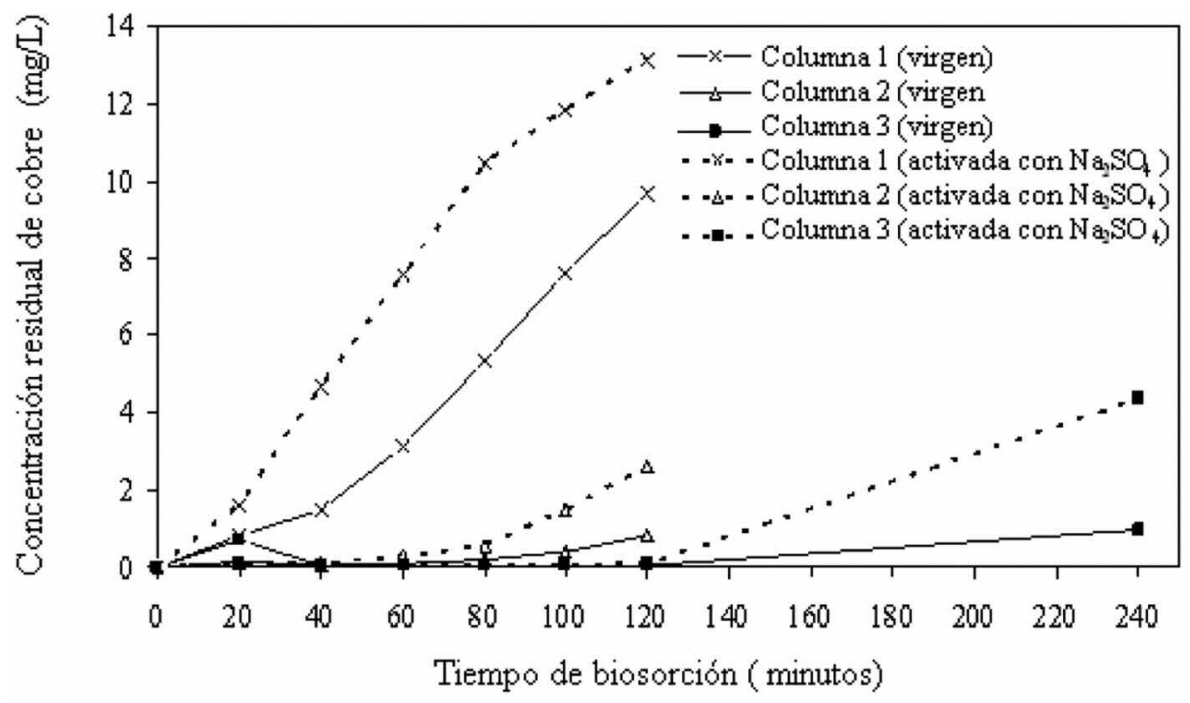

Figura 9. Concentración residual de cobre en el efluente, para cada columna del sistema, en función del tiempo de biosorción.

Figure 9. Residual concentration of copper in the effluent, for each column of the system, in function of the time of biosorption. 
para cada columna del sistema. En estos ensayos se comparó la eficacia de captación de las algas vírgenes y activadas con $\mathrm{Na}_{2} \mathrm{SO}_{4}$.

Se observa que a medida que aumenta el tiempo de biosorción se incrementa la concentración de cobre en el efluente, es decir baja la eficacia de retención de las algas. Este efecto es más notorio en la primera columna ya que retiene la mayor cantidad de iones cobre. Lo anterior es consecuencia de la disminución de sitios activos de las algas debido al aumento de los iones cobre adsorbidos en ella. Comparando ambas biomasas se observa un mejor rendimiento de las algas vírgenes en cada columna, estos resultados difieren de los obtenidos en las pruebas batch, a pesar que se ha utilizado el mismo reactivo en la activación de las algas existe diferencia en el procedimiento empleado ya que el caso de las pruebas batch se consideró el secado de las algas activadas situación que no fue contemplada en los ensayos en continuo.

Diversos investigadores han estudiado el comportamiento del proceso de biosorción en sistemas continuos entre ellos Artola et al. ${ }^{[37]}$ quienes centraron su estudio en la eliminación de cobre desde aguas residuales mediante fangos biológicos consiguiendo eliminaciones de cobre superiores a $80 \%$, mientras que Díaz et al. ${ }^{[32]}$ realizaron pruebas en continuo con flujo ascendente, utilizando algas prelavadas para la eliminación de iones hierro, aluminio y manganeso, donde este biosorbente presentó un excelente comportamiento en la eliminación de aluminio.
En la figura 10 se observan las curvas de ruptura del sistema total de columnas, para biomasas vírgenes y activadas con $\mathrm{Na}_{2} \mathrm{SO}_{4}$, en las cuales el tiempo de ruptura se ha determinado cuando la concentración final de salida del efluente es igual al $5 \%$ de la concentración inicial, y el tiempo de saturación se obtuvo cuando la concentración final es igual al $90 \%$ de la concentración inicial.

Se aprecia que cuando las algas son activadas con $\mathrm{Na}_{2} \mathrm{SO}_{4}$, se alcanza el tiempo ruptura a los $146 \mathrm{~min}$, luego se produce una rápida cinética de biosorción hasta alcanzar el tiempo de saturación a los $556 \mathrm{~min}$. Con algas vírgenes se observa un comportamiento similar en la cinética de biosorción a la obtenida con algas activadas, sin embargo los resultados obtenidos muestran un tiempo de ruptura de 240 min y un tiempo de saturación de $620 \mathrm{~min}$, lo que indica un mejor rendimiento de ésta biomasa con respecto a las algas activadas con $\mathrm{Na}_{2} \mathrm{SO}_{4}$ Vilar et al. ${ }^{[38]}$ realizaron pruebas en continuo con algas Gelidium y material compuesto como biosorbentes para remover cobre logrando tiempos de ruptura superiores a 60 y $20 \mathrm{~min}$, mientras que los tiempos de saturación corresponden a 80 y 27 min aproximadamente, tiempos que resultan ser inferiores a los obtenidos en este estudio bajo condiciones de trabajo muy similares.

En la figura 11 se muestra la variación del $\mathrm{pH}$ final en función del tiempo de biosorción, usando biomasa virgen y biomasa activada con $\mathrm{Na}_{2} \mathrm{SO}_{4}$. En el caso de la biomasa virgen se observa que el $\mathrm{pH}$ final aumenta al comienzo de la operación alcanzando un

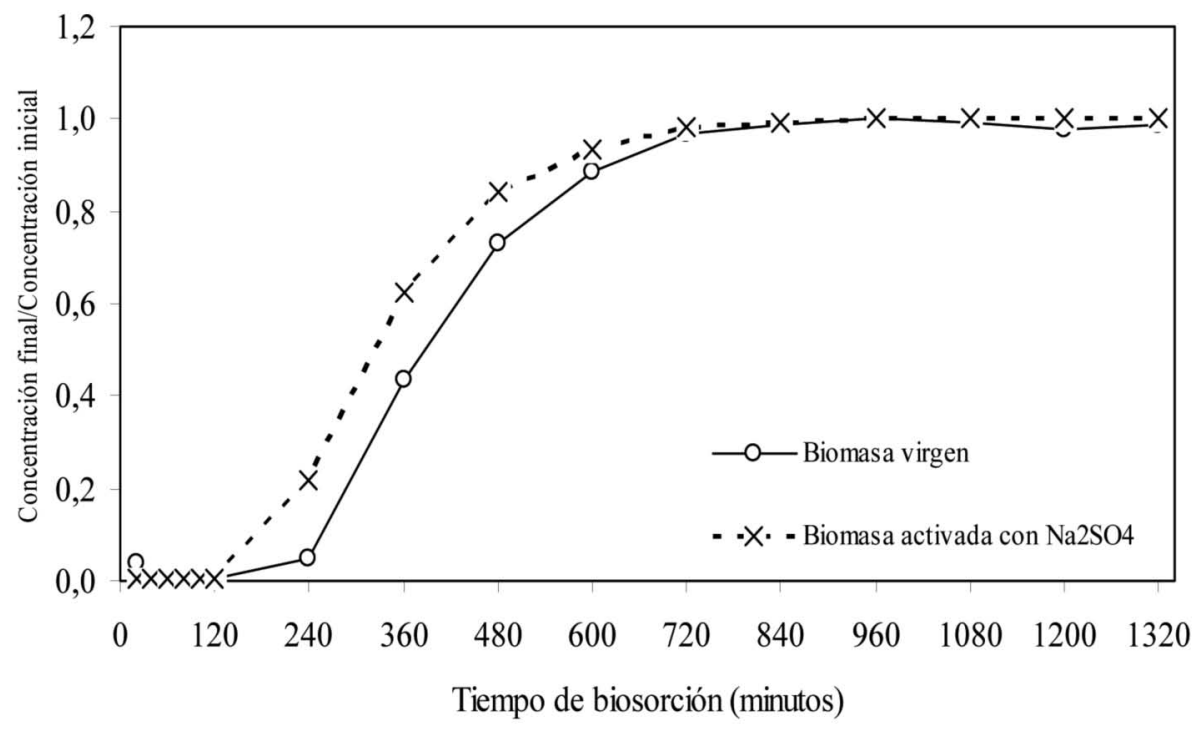

Figura 10. Curvas de ruptura del sistema de columnas para biomasas vírgenes y activadas con $\mathrm{Na}_{2} \mathrm{SO}_{4} 0,1 \mathrm{~N}$.

Figure 10. Rupture Curves of the system of columns for virgin biomasses and activated with $\mathrm{Na}_{2} \mathrm{SO}_{4} 0.1 \mathrm{~N}$. 


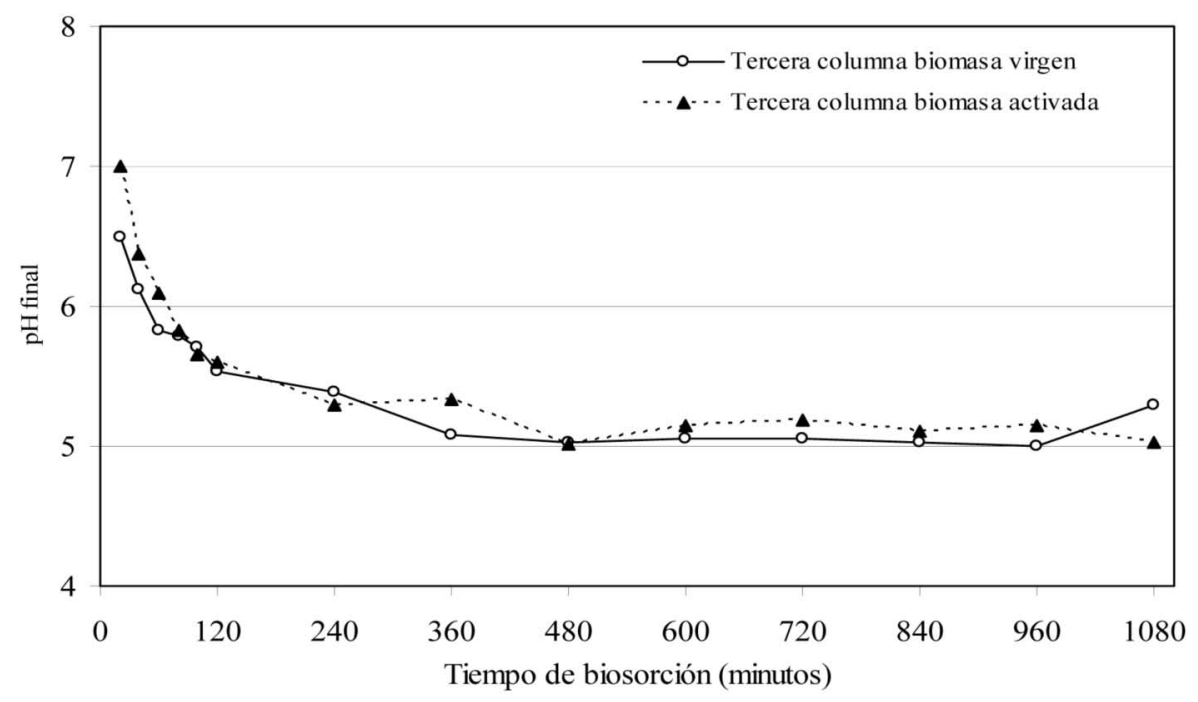

Figura 11. Variación del pH con respecto al tiempo de biosorción, usando biomasa virgen y biomasa activada con $\mathrm{Na}_{2} \mathrm{SO}_{4}$.

Figure 11. Variation of the $\mathrm{pH}$ in function of the time of biosorption, using virgin biomass and activated biomass with $\mathrm{Na}_{2} \mathrm{SO}_{4}$.

valor de 6,5, posteriormente el $\mathrm{pH}$ disminuye a medida que transcurre el tiempo de biosorción. A los 360 min de operación se alcanza prácticamente el valor inicial ( $\mathrm{pH}$ 5), manteniéndose constante hasta los $1.080 \mathrm{~min}$, donde se observa un leve incremento del $\mathrm{pH}$ a 5,3. La misma tendencia respecto del $\mathrm{pH}$ se logra con algas Gelidium [38].

En base a los resultados obtenidos, se puede señalar que existe una relación entre la variación de $\mathrm{pH}$ y la captación de cobre, es decir, al principio de la operación la captación de cobre es más elevada produciéndose un aumento en el $\mathrm{pH}$ final, posteriormente, al disminuir el pH la captación de cobre baja. Sin embargo, cuando se alcanza la saturación el pH tiende a elevarse provocando una leve disminución en la captación de cobre. Estos resultados pueden estar justificados por la posible retención de iones $\mathrm{H}^{+}$en las algas, lo que aumentaría el pH del medio. Así, a medida que el alga se va saturando, disminuye la eficacia de retención y se incrementa el $\mathrm{pH}$, tendiendo a su valor inicial.

\subsubsection{Efecto de la altura del lecho}

Para determinar el efecto de la altura del lecho sobre la biosorción de cobre, se utilizó solamente biomasa activada con $\mathrm{Na}_{2} \mathrm{SO}_{4}$, evaluándose tres alturas de lecho en columnas de $4,5 \mathrm{~cm}$ de diámetro, las masas de biosorbente corresponden a: $25 \mathrm{~g}(25 \mathrm{~cm}) ; 50 \mathrm{~g}$
$(50 \mathrm{~cm}) ; 75 \mathrm{~g}(75 \mathrm{~cm})$. Los resultados de estas pruebas se muestran en la figura 12, en la cual se observa un efecto positivo en la eliminación de cobre cuando se incrementa la altura del lecho, permitiendo de esta manera alcanzar la saturación del biosorbente a tiempos superiores a los $120 \mathrm{~min}$, las captaciones de cobre para cada una de las alturas consideradas (25, 50 y $75 \mathrm{~cm}$ ) corresponden a 90,9; 98,9 y 99,7\%, también se observa que el tiempo de ruptura crece a medida que aumenta la altura del lecho, el caudal de alimentación de la solución fue igual a $50 \mathrm{ml} / \mathrm{min}$,

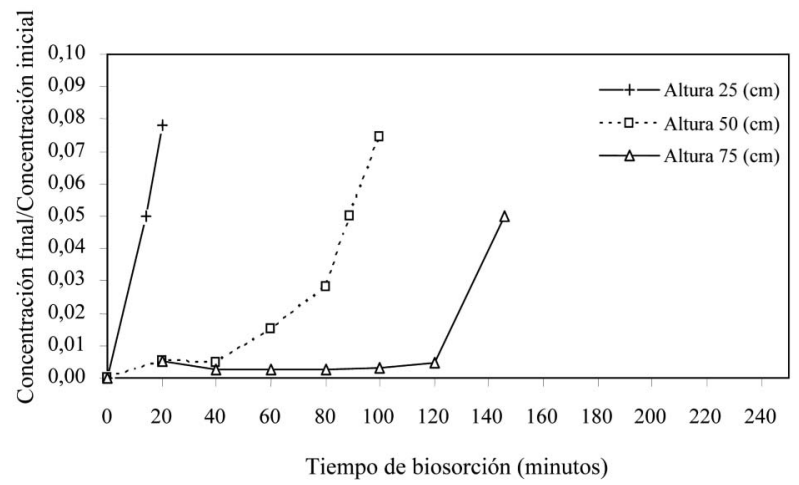

Figura 12. Efecto de la altura del lecho sobre la biosorción de iones cobre.

Figure 12. Height of bed effect on the biosorption of ions copper. 
al cual le corresponde una velocidad lineal de $3,14 \mathrm{~cm} / \mathrm{h}$ (porosidad de lecho de $93 \%$ ), Hasan y Srivastava ${ }^{[39]}$ evaluaron el efecto de la altura del lecho a distintas velocidades para soluciones que contienen inicialmente $50 \mathrm{mg} / \mathrm{l}$ de cobre consiguiendo eliminaciones cercanas a $90 \%$ para una altura de $20 \mathrm{~cm}$ cuando la velocidad lineal de la solución fue de $3,185 \mathrm{~cm} / \mathrm{h}$.

Los resultados obtenidos, se examinan relacionando el tiempo de servicio o ruptura con la altura del lecho mediante el modelo BDST, presentado en la ecuación 1 . Si se representan los valores del tiempo de ruptura frente a la altura del lecho, $\mathrm{Z}$, la pendiente y la ordenada en el origen permiten determinar los parámetros del modelo (Fig. 13).

$$
t_{r}=\frac{N_{0} Z}{C_{i} V}-\frac{1}{k_{a} \cdot C_{i}} \operatorname{Ln}\left(\frac{C_{i}}{C_{f}}-1\right)
$$

Donde " $t_{r}$ " es el tiempo de ruptura (min), " $N_{0}$ " es la capacidad de sorción máxima del lecho (mg/l), "V" es la velocidad lineal $(\mathrm{cm} / \mathrm{min})$, " $k_{a}$ " es la constante de velocidad en el lecho ( $1 / \mathrm{mg} \cdot \mathrm{min})$, " $\mathrm{C}_{i}$ y $\mathrm{C}_{f}$ " corresponden a las concentraciones iniciales y finales del efluente respectivamente $(\mathrm{mg} / \mathrm{l})$.

Con la aplicación del modelo BDST se obtiene un coeficiente de correlación igual a 0,9938, lo cual indica un buen ajuste, obteniéndose una capacidad de sorción del lecho $\left(N_{0}\right)$ igual a $136 \mathrm{mg} / \mathrm{l}$ que es concordante con los $127 \mathrm{mg} / \mathrm{l}$ conseguidos por Hasan et al. ${ }^{[39]}$, para condiciones de concentración inicial de cobre de $50 \mathrm{mg} / \mathrm{l}$ el cual fue alimentado a una velocidad de $3,185 \mathrm{~cm} / \mathrm{h}$, mientras que la constante de velocidad $\left(K_{a}\right)$ alcanza un valor igual a $2,67 \cdot 10^{-3} \mathrm{l} / \mathrm{mg} \cdot \mathrm{min}$. Sustituyendo los valores de los parámetros en la ecuación 1, se obtiene la siguiente expresión (ecuación 2);

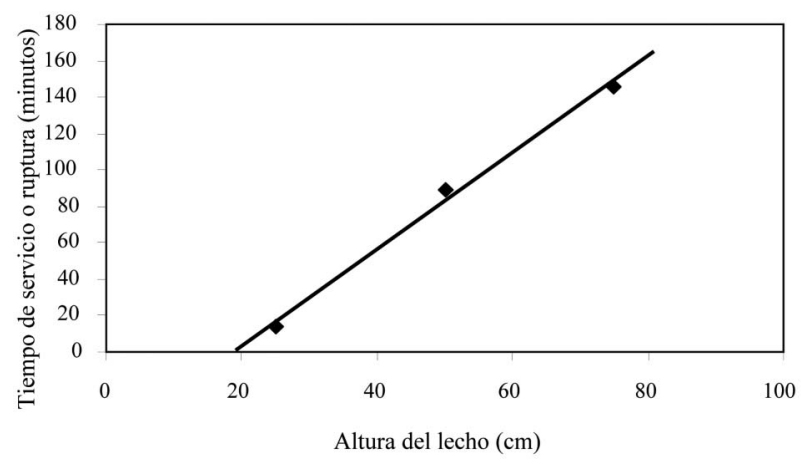

Figura 13. Tiempos de ruptura para tres distintas alturas de lecho.

Figure 13. Times of rupture for three different bed heights.

$$
t_{r}=\frac{136 Z}{C_{i} V}-\frac{1}{\left(2,67 \cdot 10^{-3}\right) \cdot C_{i}} \operatorname{Ln}\left(\frac{C_{i}}{C_{f}}-1\right)
$$

En la tabla I se muestran los valores del tiempo de ruptura obtenidos experimentalmente y los calculados a partir de la ecuación 2. Comparando los datos experimentales con los valores calculados, se observa una mínima diferencia, esto se debe al buen ajuste de la curva.

La profundidad crítica del lecho que corresponde a la profundidad teórica suficiente para que en $\mathrm{t}=0$ la concentración de soluto en el efluente tratado exceda la concentración de ruptura. Para determinar este parámetro de diseño se considera $t_{\mathrm{r}}=0 \mathrm{y}$ se reemplaza en la ecuación (2), obteniéndose el valor de la altura crítica, $Z_{0}=19(\mathrm{~cm})$, con respecto a las alturas de lecho analizadas. Así, para cada columna se necesitará una altura de $6(\mathrm{~cm})$ aproximadamente para alcanzar la ruptura al inicio de la biosorción.

Vijayaraghavan et al. ${ }^{[40]}$ también aplicaron el modelo BDST para la adsorción de iones cobre en algas marrón oscuro obteniendo un muy buen ajuste del modelo con los datos experimentales $\left(R^{2}=0,999\right)$, mostrando la validez del modelo para el sistema estudiado. Estos investigadores señalan que el modelo BDST puede ser útil para el escalamiento del proceso, opinión que concuerda con el ajuste logrado por Hasan et al. ${ }^{[39]}$ cuyos valores experimentales muestran un ajuste cuyo coeficiente de correlación es igual a 0,999.

\subsubsection{Ensayos de desorción en columnas}

La regeneración y reutilización de los materiales biosorbentes también debe considerarse al evaluar la eficacia y la viabilidad del proceso de biosorción. Si el agente activo puede ser regenerado a través de un

Tabla I. Valores del tiempo de rupturas obtenidas en forma experimental y calculada

Table I. Values of experimental and calculated rupture times

\section{Z (cm) Tiempo de ruptura ( $\mathrm{min})$ \\ Experimental Calculado}

$\begin{array}{rrr}25 & 14 & 17 \\ 50 & 89 & 83 \\ 75 & 146 & 149\end{array}$


ciclo de desorción sin destruir la integridad de la pared celular el proceso será más lucrativo ${ }^{[41]}$. Es por esto que se efectuaron pruebas para evaluar el comportamiento de las algas activadas con $\mathrm{Na}_{2} \mathrm{SO}_{4}$ en un segundo ciclo de biosorción utilizándose una solución de $\mathrm{HCl}$ para efectuar la desorción de los iones cobre adsorbidos en el primer ciclo, la solución alimentada al sistema de columnas en ambos ciclos contenía 20,35 mg/l de cobre y pH 5,01.

En la figura 14, se observan las curvas de rupturas obtenidas para el primer y segundo ciclo de biosorción. En el segundo ciclo de biosorción se alcanzan mejores resultados, con un tiempo de ruptura de 307 min y un tiempo de saturación de 903 min, comparados con los resultados del primer ciclo de biosorción (algas sin regenerar), en el cual se obtiene un tiempo de ruptura de 146 min y un tiempo de saturación de $556 \mathrm{~min}$. Los resultados del segundo ciclo dejan de manifiesto, que el poder de captación del biosorbente se ve favorecido al efectuar la desorción de cobre con la solución de $\mathrm{HCl}$. Este comportamiento puede explicarse en función de la caracterización iónica de las algas las cuales contienen $\mathrm{Na}^{+}(664 \mathrm{mg} / \mathrm{l}), \mathrm{Ca}^{+}(339 \mathrm{mg} / \mathrm{l}), \mathrm{K}^{+}(724 \mathrm{mg} / \mathrm{l})$ y $\mathrm{Mg}^{+}(300 \mathrm{mg} / \mathrm{l})$ los que también son retirados desde el biosorbente cuando se realiza la desorción del cobre después de concluido el primer ciclo, por lo que al efectuar nuevamente la activación con $\mathrm{Na}_{2} \mathrm{SO}_{4}$ del biosorbente existirá mayor presencia de iones $\mathrm{Na}^{+}$disponibles para intercambiarse con el cobre provocando un aumento de los sitios activos disponibles para la biosorción.

Utilizando una solución de $\mathrm{HCl} \mathrm{0,2M,} \mathrm{para} \mathrm{remo-}$ ver iones cobre y níquel desde algas en pruebas batch Basha et al. ${ }^{[8]}$, en cinco ciclos sucesivos muestran que la capacidad de sorción de las biomasas por cobre y níquel permanecen relativamente constante en todos los ciclos, demostrando que la biomasa puede ser regenerada completamente.

Vilar et al..$^{[38]}$, en un sistema de columnas empacadas con algas Gelidium, desorbieron el cobre con soluciones $0,1 \mathrm{M}$ de ácido nítrico $\left(\mathrm{HNO}_{3}\right)$ y posteriormente éstas fueron utilizadas en un nuevo ciclo de biosorción, según los resultados demuestran que la capacidad de captación de iones cobre de la biomasa permanece constante en los dos ciclos evaluados, por lo que la estructura celular no sufrió daños durante la regeneración. De igual forma Hasan et al. ${ }^{[39]}$ empleando biomasa inmovilizada efectuaron 6 ciclos de sorción/desorción, no existiendo consenso respecto del comportamiento de la biomasa en los ciclos sucesivos de regeneración, ya que ellos observan disminuciones en la eliminación de cobre en los ciclos posteriores, a pesar de esto indican que el $\mathrm{HCl}$ es eficaz en la regeneración del biosorbente.

El desempeño del diferentes desorbentes $\left(\mathrm{HNO}_{3}\right.$, $\mathrm{Ca}\left(\mathrm{NO}_{3}\right)_{2}$ y EDTA) de iones cobres fue llevada a cabo por Deng et al. ${ }^{[11]}$, presentando el EDTA los mejores resultados en la regeneración causando una

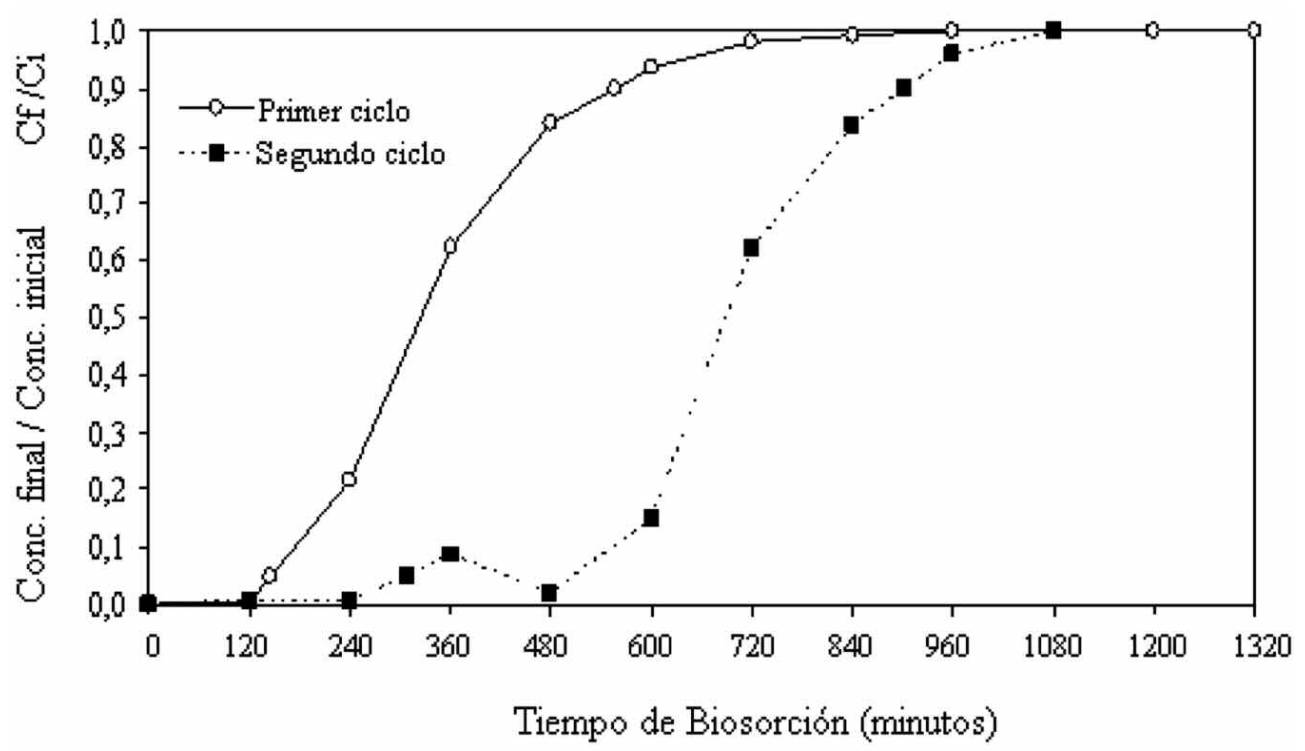

Figura 14. Comparación de las curvas de ruptura de los ensayos para el primer y segundo ciclo de biosorción.

Figure 14. Comparison of the rupture curves of the tests for the first and second cycle of biosorption. 
pérdida igual a $10 \%$ en la capacidad de biosorción de algas Cladophora fascicularis.

Volesky et al. ${ }^{[42]}$ trabajando con Sargassum filipéndula como biosorbente de iones cobre, realizan la regeneración con $\mathrm{CaCl}_{2} / \mathrm{HCl}$ en $\mathrm{pH} 3$ obteniendo eficacias de elusión de hasta $100 \%$. Por otra parte Vegliò et al. ${ }^{[31]}$ efectúan la regeneración de residuos de aceituna con soluciones ácidas alcanzándose eliminaciones de cobre de alrededor de $40 \%$ en las mismas condiciones experimentales de los ensayos de la primera adsorción. Estos investigadores señalan que la regeneración de los residuos con diferentes concentraciones de EDTA presenta una disminución de sitios activos de adsorción. Ellos concluyen que el uso de $\mathrm{HCl}_{\text {y }} \mathrm{CaCl}_{2}$ permite regenerar completamente el material biosorbente.

\section{CONCLUSIONES}

Fue investigado el desempeño de las biomasas algas verdes y orujos en la biosorción de cobre desde soluciones acuosas. Las siguientes conclusiones pueden deducirse de este estudio:

- El aumento del $\mathrm{pH}$ presenta un aumento en la captación de iones cobre alcanzando eliminaciones máximas ( $\approx 54 \%$ ) en el rango de $\mathrm{pH}$ entre 4 y 6 , para algas verdes, mientras que el orujo no presenta grandes diferencias en la captación de cobre con el aumento del $\mathrm{pH}$, donde la eliminación para el rango de $\mathrm{pH}$ estudiado varia entre $17,5 \%$ a $22,5 \%$.

- La cinética de biosorción se produce en forma rápida durante los primeros $5 \mathrm{~min}$, logrando eliminaciones cercanas a $48 \%$ para las algas y $16 \%$ para el orujo, a tiempos mayores en ambos casos no se logra incrementar significativamente la eliminación de cobre, los valores máximos correspondientes, es decir el tiempo óptimo, se estima igual a $15 \mathrm{~min}$.

- Las bajas eliminaciones alcanzadas con los orujos (desechos de aceitunas) indican que no es un sorbente adecuado para remover iones de cobre. Debido a que se consiguió solamente un $25 \%$ de eliminación con ellos.

- El proceso de activación química incrementa en un $35 \%$ la capacidad de biosorción de las algas y en un $50 \%$ la de los orujos. Los mejores resultados se obtienen con $\mathrm{Na}_{2} \mathrm{SO}_{4}$, alcanzándose eliminaciones superiores al $96 \%$ con las algas y mayores al $55 \%$ con los orujos.

- Las algas demuestran gran efectividad en la retención de cobre, obteniéndose un $62 \%$ y un $96 \%$ de eliminación de $\mathrm{Cu}^{+2}$ en ensayos batch, usando algas vírgenes y algas activadas con $\mathrm{Na}_{2} \mathrm{SO}_{4}$ respectivamente, por lo que este agente activador provoca un aumento de los sitios disponibles para la captación de cobre.

- En las pruebas realizadas en un sistema continuo, las algas vírgenes muestran gran eficacia en la captación de iones cobre, superando a la captación alcanzada con las algas activadas con $\mathrm{Na}_{2} \mathrm{SO}_{4}$.

- El uso de una solución de $\mathrm{HCl}$ para desorber cobre desde las algas no provoca daños al biosorbente, afectando positivamente su capacidad de extracción de cobre en un segundo ciclo de biosorción.

- Las algas activadas con $\mathrm{Na}_{2} \mathrm{SO}_{4}$ muestran buena eficacia en la eliminación de cobre al ser expuestas a un segundo ciclo de biosorción, lo que indica que pueden ser regeneradas y reutilizadas en un segundo ciclo.

- El aumento de la altura del lecho provoca un efecto positivo en la eliminación de cobre y un incremento del tiempo en el que se consigue la saturación.

- Mediante el modelo BDST, se obtuvo una expresión que puede ser aplicada con otras condiciones de trabajo. Además, se calculó la altura crítica del lecho, parámetro útil para el diseño de equipos y para otras experiencias.

- Finalmente, se puede señalar que las algas tienen alta aplicabilidad en la biosorción de iones cobre, debido a que es un sorbente eficiente en la retención de este metal. Además de ser un biosorbente económico y de fácil obtención, también pueden ser regeneradas y reutilizadas en una segunda operación de biosorción, lo que hace económico y rentable el proceso.

\section{Agradecimientos}

Los autores agradecen el apoyo a la Dirección de Investigaciones de la Universidad de Atacama por el financiamiento del proyecto 221156 DIUDA 06/18, del cual forma parte este trabajo, y agradecen la colaboración del Sr. Bruno Zazzali por su apoyo en el desarrollo experimental.

\section{REFERENCIAS}

[1] B. Atkinson, F. Bux y H. Kasan, Water SA. 24 (1998) 151-164.

[2] S. Hidalgo, Tesis Doctoral, Universidad Politécnica de Cataluña, Barcelona, 2004. 
[3] S. Lee, C. Jung, H. Chung, M. Lee y J.Yang, Process Biochemistry, 33(1998) 205-211.

[4] S. Deng e Y. Ting, Water Res. 39 (2005) 2.1672.177 .

[5] D. Zhou, L. Zhang y S. Guo, Water Res. 39 (2005) 3.755-3.762.

[6] M. Santarder, P. Tapia, O. Pavez, L. Valderrama y D. Guzman, Rev. Metal. Madrid 45 (2009) 365-374.

[7] T. Davis, B. Volesky y A. Mucci, Water Res. 37(2003) 4.311-4.330.

[8] S. Basha, Z. V. P. Murthy y B. Jha, Ind. Eng. Chem. Res. 48 (2009) 961-975.

[9] V. Murphy, H. Hughes y P. Mcloughlin, Water Res. 41(2007) 731-740.

[10] G. Naja y B. Volesky, Colloids and Surfaces A: Physicochem. Eng. Aspects, 281(2006)194-201.

[11] B. Jha, S. Basha, S. Jaiswar, B. Mishra y M.C. Thakur, Biodegradation 20 (2009)1-13.

[12] G. Huamán y M.L. Torem, PERUMIN, 29 Convención Minera, Arequipa, Perú (2009).

[13] F. Luo, Y. Liu, X. LI, Z. Xuan y J. MA, Chemosphere 64 (2006) 1.122-1.127.

[14] O. Demirbas, A. Karadag, M. Alkan y M. Dogan, J. Hazard. Mater. 153 (2008) 677684.

[15] M. Dundar, C. Huhoglu e Y. Nuhoglu, J. Hazard. Mater. 151 (2008) 86-95.

[16] W. Zheng, X. LI, F. Wang, Q. Yang, P. Deng y G. Zeng, J. Hazard. Mater. (2008), article in press, doi: 10.1016/j.jhazmat.2008.01.029.

[17] T. Altun y E. Pehlivan, Clean 35 (2007) 601606.

[18] S. Lee y J. Yang, Separ. Sci. Technol. 32 (1997) 1.371-1.387.

[19] Q. Yu y P. Kaewsarn, Separ. Sci. Technol. 34 (1999) 1.595-1.605.

[20] D. Kratochvil y B. Volesky, Water Research. 32 (1998) 2.760-2.768.

[21] L. Deng, X. Zhu, X. Wang, Y. Su y H. Su, Biodegradation 18 (2007) 393-402.

[22] F. Pagnanelli, S. Mainelli, S. De Angelis y L. Toro, Water Res. 39 (2005) 1.639-1.651.

[23] F. Pagnanelli, S. Mainelli, F. Veglio y L. Toro, Chem. Eng. Sci. 58 (2003) 4.709-4.717.

[24] M. Sarioglu, U. Asli (ATAY) Güler y N. Beyazit, Desalination 239 (2009) 167-174.
[25] A. Grimm, R. Zanzi, E. Björnbom y A. Cukierman, Bioresource Technol. 99 (2008) 2.559. 2.565 .

[26] H. Benaissa y M.A. Elouchdi, IWTC9, Ninth International Water Technology Conference, Sharm El-Sheikh, Egypt, 2005, pp. 69-83.

[27] S.K. Papageorgiou, F.K. Katsaros, E.P. Kouvelos, J.W. Nolan, H.L. Deit y N.K. Kanellopoulos, J. Hazard. Mater. B 137 (2006) 1.765-1.772.

[28] Volesky, Hydrometallurgy 71 (2003) 179-190.

[29] T. A. Davis, B. Volesky y R. H. Vieira, Water Res. 37 (2003) 4.311-4.330.

[30] N. Fiol, I. Villaescusa, M. Martinez, N. Miralles, J. Poch y J. Serarol, Separ. Sci. Technol. 50 (2006) 132-140.

[31] F. Vegliò, F. Beolchini y M. Prisciandaro, Water Res. 37(2003) 4.895-4.903.

[32] A. Diaz, J. Arias, G. Gelves, A. Maldonado, D. Laverde, J. Pedraza y H. Escalante, Revista Facultad de Ingeniería, Universidad de Antioquia, 030 (2003) 34-48.

[33] L. Sheng-Lian, Y. Lin, CH. Li-Yuan, M. XiaoBo, W. Yun-Yan, F. Yan y W. Pu, Trans. Nonferrous Met. Soc. China 16 (2006) 1.4311.435 .

[34] P. Lodeiro, B. Cordero, Z. Grille, R. Herrero y M.E. Sastre DE Vicente, Biotechnol. Bioeng. 88 (2004) 237-247.

[35] M.A. Martin-Lara, F. Pagnanelli, S. Mainelli, M. Calero y L. Toro, J. Hazard. Mater. 156 (2008) 448-457.

[36] F. Pagnanelli, S. Mainelli y L. Toro, Water Res. 42 (2008) 2.953-2.962.

[37] A. Artola, M.J. Martín, M.D. Balaguer y M. Rigola, Ingeniería Química Tratamiento de Aguas Residuales (2003) 180-184.

[38] V.J.P. Vilar, C.M.S. Botelho, J.M. Loureiro y R.A.R. Boaventura, Bioresource Technol. 99 (2008) 5.830-5.838.

[39] S. H. Hasan y P. Srivastava, J. Environ, Manage. 90 (2009) 3.313-3.321.

[40] K. Vijayaraghavan, J. Jegan, K. Palanivelu y M. Velan, Chem. Eng. J. 106 (2005) 177-184.

[41] B.W. Atkinson, F. Bux y H.C. Kasan, Water SA. 24 (1998) 129-136.

[42] B. Volesky, J. Weber y J. Park, Water Res. 37(2003) 297-306. 